\title{
Modelling of propane emissions from a tank containing a liquefied phase
}

\author{
Zdzisław Salamonowicz ${ }^{1, *}$ and Radosław Makowski ${ }^{1}$ \\ ${ }^{1}$ Faculty of Fire Safety Engineering, The Main School of Fire Service, Słowackiego 52/54 St., 01-629 Warsaw, Poland
}

\begin{abstract}
During gas-phase release from the tank containing liquefied gas, the phenomenon of boiling, heat transfer from moist air to the tank, heat exchange between shell, liquid, and vapor, and mass loss can be observed. The aim of this paper is modelling the thermal response of the tank containing liquefied gas during jet emission of the vapor phase. The model takes into account heat exchange between air, tank's shell, liquid phase, vapor phase and mass balance. The proposed model predicts: pressure inside the tank, tank's shell temperature in part with liquid and vapor, the temperature of the liquid phase and vapor phase, and mass loss from the tank. The results of the theoretical model were compared with experimental results. An experiment based on the typical home container for LPG, containing $10 \mathrm{~kg}$ of propane ( $27 \mathrm{dm}^{3}$ volume) was conducted. In general, the proposed model predicts well the changes in measured parameters temperatures and pressure - during vapor phase emission from a tank containing liquefied gas.
\end{abstract}

\section{Introduction}

The process of storing and transporting liquefied gases in pressure tanks, from the safety point of view, requires the development of emergency scenarios and risk analysis. Storage tanks for liquefied medium under pressure can be found both in the refinery, energy and transport industries. A special way, as an alternative to natural gas, is the use of propane-butane in home tanks and portable cylinders.

The analysis of phenomena during the opening of a safety valve in a tank containing a liquefied medium is conducted by many researchers $[1-7,11]$ and is a complex issue requiring the consideration of many variables [8-12]. During the sudden opening of the safety valve or damage to the tank in the upper part, the vapor phase is emitted with the simultaneous process of the liquid boiling. Due to the low boiling point, this process can be very intensive, even with two-phase emission [23]. The modelling of the evaporation of the vapor phase from the tank is based on the mass and energy balance of the liquid-vapor-tank-environment system. The most important issues are related to heat exchange with the environment including condensation of steam with further freezing and mass exchange related to the boiling process and emission through the outlet.

\section{Modelling and experiments}

\subsection{Model}

The proposed model of jet outflow of propane from the tank presents changes in thermodynamic parameters during emergency release. The model allows to simulate the change of the following system parameters: temperature of the gas phase, temperature of the liquid phase, temperature of the upper part of the tank wall (in contact with the gas phase), temperature of the lower part of the tank wall (in contact with the liquid phase), mass of the gas phase and mass of the liquid phase. The model analyses the change of the above parameters during jet outflow and immediate ignition. The isentropic-adiabatic discharge takes place through an outlet of a given diameter. The algorithm, depending on the pressure in the tank, includes the throttled and unthrottled flow. Numerical calculations were made using Matlab's - mathematical computation environment.

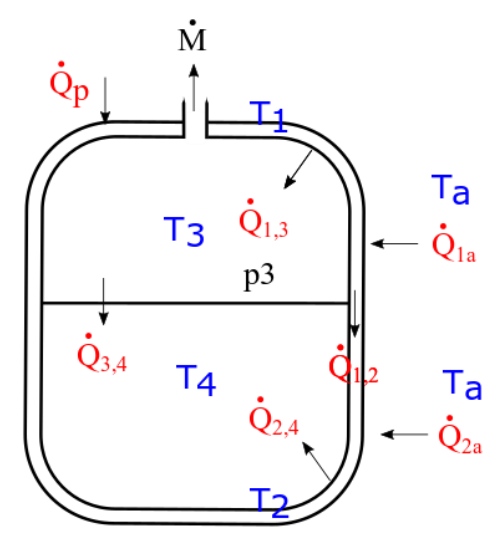

Fig. 1. Propane-tank-environment system.

\footnotetext{
*Corresponding author: zsalamonowicz@sgsp.edu.pl
} 
Boundary conditions:

- in the initial stage, the liquid phase and the gas phase are in equilibrium,

- the tank-environment system is also in equilibrium, i.e. the initial temperatures: liquid phase, gas phase, tank (both upper and lower part) are equal to the ambient temperature,

- the ambient temperature is constant,

- the flow of heat flux through the walls of the tank is treated as flow through an infinitely thin shell (with the flow of heat the thickness of the tank is not taken into account),

- in calculations, the tank is treated as a homogeneous cylinder,

- the tank's shell has been divided into two parts by an imaginary line, reflecting the contact of the lower and upper parts of the tank with the liquid phase and the gas phase respectively,

- temperatures of the: liquid phase, gas phase, lower and upper part of the tank's shell are constant in the entire volume,

- coefficients used in the model are treated as constant values, their changes are not including changes of the thermodynamic parameters,

- values which are treated as variables in time are: heat exchange coefficients, mass streams: outflow and evaporation, liquid and gas phase density, height of both phases, heat exchange surfaces, heat flux from the environment, heat flux delivered from a fire, heat flux of liquid phase vaporization, flux of heat discharged into the environment from the gas phase, pressure in the tank.

The analysis of parameter changes over time results from the solution of the following system of equations:

$$
\left\{\begin{array}{c}
Q_{1}=\dot{Q}_{1 a} t-\dot{Q}_{1,3} t-\dot{Q}_{1,2} t+\dot{Q}_{p} t \\
Q_{2}=\dot{Q}_{2 a} t+\dot{Q}_{1,2} t-\dot{Q}_{2,4} t \\
Q_{3}=\dot{Q}_{1,3} t-\dot{Q}_{3,4} t-\dot{Q}_{s t r} t \\
Q_{4}=\dot{Q}_{2,4} t+\dot{Q}_{3,4} t-\dot{Q}_{l g} t \\
m_{3}=m_{30}-\dot{M}_{s t r} t+\dot{M}_{l g} t \\
m_{4}=m_{40}-\dot{M}_{l g} t
\end{array}\right.
$$

which considered in detail takes the form:

$$
\left\{\begin{array}{c}
\frac{d T_{1}}{d t}=\frac{h_{1 a} A_{1 a}\left(T_{a}-T_{1}\right) t-h_{1,3} A_{1,3}\left(T_{1}-T_{3}\right) t-h_{s t} A_{s t}\left(T_{1}-T_{2}\right) t+\dot{Q}_{p} t}{m_{1} c_{s t}} \\
\frac{d T_{2}}{d t}=\frac{h_{2 a} A_{2 a}\left(T_{a}-T_{2}\right) t+h_{s t} A_{s t}\left(T_{1}-T_{2}\right) t-h_{2,4} A_{2,4}\left(T_{2}-T_{4}\right) t}{m_{2} c_{s t}} \\
\frac{d T_{3}}{d t}=\frac{h_{1,3} A_{1,3}\left(T_{1}-T_{3}\right) t-h_{3,4} A_{3,4}\left(T_{3}-T_{4}\right) t-\dot{Q}_{s t r} t}{m_{3} c_{3}} \\
\frac{d T_{4}}{d t}=\frac{h_{2,4} A_{2,4}\left(T_{2}-T_{4}\right) t+h_{3,4} A_{3,4}\left(T_{3}-T_{4}\right) t-\dot{Q}_{l g} t}{m_{4} c_{4}} \\
\frac{d m_{3}}{d t}=-\dot{M}_{s t r} t+\dot{M}_{l g} t \\
\frac{d_{m 4}}{d t}=-\dot{M}_{l g} t
\end{array}\right.
$$

The mass flow rate (mstr) was calculated including the throttled outflow criterion according to the formula [27]:

$$
p_{3} \geq p_{a}\left(\frac{\gamma+1}{2}\right)^{\frac{\gamma}{\gamma-1}}
$$

The throttled outflow was calculated from the equation:

$$
\dot{M}_{s t r}=A_{h} p_{3}\left[\frac{y M}{R T_{3}}\left(\frac{2}{y+1}\right)^{\frac{y+1}{y-1}}\right]^{\frac{1}{2}}
$$

whereas the unthrottled outflow was calculated by the equation:

$$
\dot{M}_{\text {str }}=A_{h} \sqrt{2 \rho_{3} p_{3}\left(\frac{y}{y-1}\right)\left[\left(\frac{P_{a}}{p_{3}}\right)^{\frac{2}{y}}-\left(\frac{P_{a}}{p_{3}}\right)^{\frac{y+1}{y}}\right]}
$$

The heat flux from the jet fire is described by the formula [24]:

$$
\dot{Q}_{p}=\frac{A_{34} \eta \tau_{a} \dot{M}_{s t r} H_{c o m b}}{4 \pi x^{2}}
$$

The heat flow needed to evaporate the boiling liquid is calculated on the basis [21]:

$$
\dot{Q}_{l g}=A_{34} \mu_{4} h_{l g}\left[\frac{\left(\rho_{4}-\rho_{3}\right) g_{z}}{\sigma}\right]^{0,5}\left[\frac{C_{p 4}\left(T_{2}-T_{s a t}\right)}{c_{s f} h_{l g} P r_{l}^{n}}\right]^{3}
$$

The mass flow of evaporation:

$$
\dot{M}_{l g}=\frac{\dot{Q}_{l g}}{h_{l g}}
$$

The initial masses of gaseous and liquid phases were calculated from the system of equations:

$$
\left\{\begin{array}{l}
m_{30}+m_{40}=m_{\text {netto }} \\
\frac{m_{30}}{\rho_{30}}+\frac{m_{40}}{\rho_{40}}=\pi r^{2} H_{z b}
\end{array}\right.
$$

The Peng-Robinson equation [28] was used to calculate the liquid-vapor balance:

$$
p=\frac{R T}{V-b}-\frac{a \propto(T)}{V(V+b)+b(V-b)}
$$

Heat exchange coefficients between the environment and the upper and lower tank parts were calculated from equations [26]:

$$
\begin{aligned}
& h_{1 a}=1,31\left(T_{1}-T_{a}\right)^{\frac{1}{3}} \\
& h_{2 a}=1,31\left(T_{2}-T_{a}\right)^{\frac{1}{3}}
\end{aligned}
$$

The heat exchange coefficient between the tank wall and the gas phase was calculated based on the equation [29]:

where:

$$
h_{13}=\frac{k_{3} C R a_{3}{ }^{\frac{1}{3}}}{H_{g}}
$$

$$
R a_{3}=\frac{g_{z} \beta_{3}\left(T_{1}-T_{3}\right) H_{g}^{3}}{\alpha_{3} v_{3}}
$$

and the coefficient of volumetric thermal expansion of the gas phase:

$$
\beta_{3}=\frac{2}{T_{1}+T_{3}}
$$

Similarly, for the heat exchange coefficient between the tank wall and the liquid phase, the following equation was used:

$$
h_{2,4}=\frac{k_{4} C R a_{4}{ }^{\frac{1}{3}}}{H_{C}}
$$

The coefficient of heat exchange along the steel wall [27]:

$$
h_{s t}=\frac{k_{s t}}{\sqrt{k_{s t} g}\left(\frac{1}{\sqrt{h_{1 a}+h_{13}}}+\frac{1}{\sqrt{h_{2 a}+h_{24}}}\right)}
$$

The heat transfer coefficient between the liquid and gas phases was calculated from the relationship between the number of Nussetel, Jakob and Prandtl [27]:

$$
h_{3,4}=\frac{k_{4} \cdot J a^{2}}{d \cdot C_{m b^{3}} \cdot \operatorname{Pr}^{l}}
$$




\subsection{Experiments}

Standard cylinders with a water capacity of $27 \mathrm{dm}^{3}$ containing $10 \mathrm{~kg}$ of propane were used for the tests. A head with a valve and inputs for the measurement elements was designed and made, in order to insert a thermocouple and a pressure sensor inside the cylinder. During the experiments, the temperature of the liquid phase, vapor phase, temperature of the outer part of the tank wall corresponding to the liquid and vapor phase, the pressure in the tank, the mass of the system and the outside temperature were recorded. Figure 2 shows the research stand in terms of visible and infrared radiation.

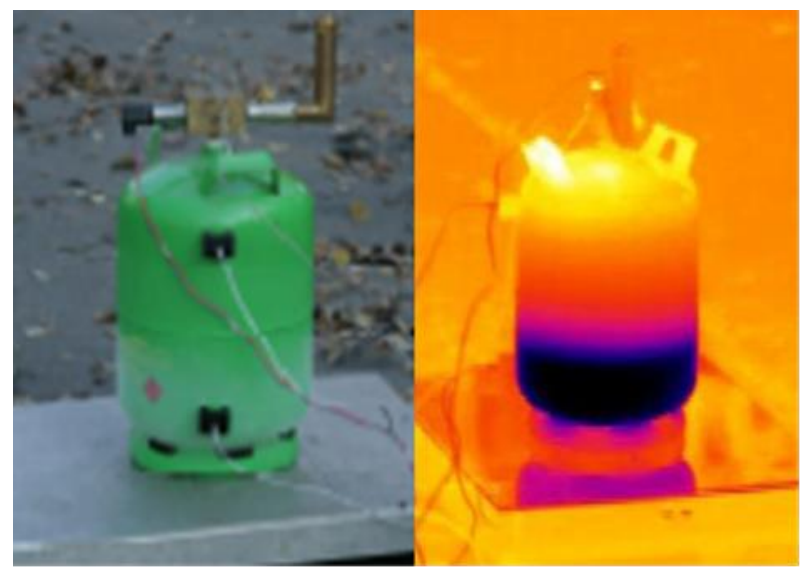

Fig. 2. Research stand - VIS and IR image.

\section{Results and discussion}

During the outflow of the gas phase from the system which was in equilibrium, the pressure was decreasing in the tank. At lower pressure, the saturation temperature is also lower, as a result of the pressure drop, the liquid is at a temperature above the saturation temperature and starts to boil. The boiling intensity is proportional to the difference between the temperature of the liquid phase and the saturation temperature at the specific pressure. The liquid phase absorbs heat from the system while evaporating. The heat absorbed by the system from the environment is lower than the heat needed to evaporate the liquid phase. Therefore, during the jet outflow, the decrease of temperature in both phases and both parts of the tank can be observed. Figures 3-6 present comparisons of changes in individual temperatures obtained from the proposed model and experimental data. The results are shown for four diameters of the outlet: $6,5,4$ and $2 \mathrm{~mm}$.

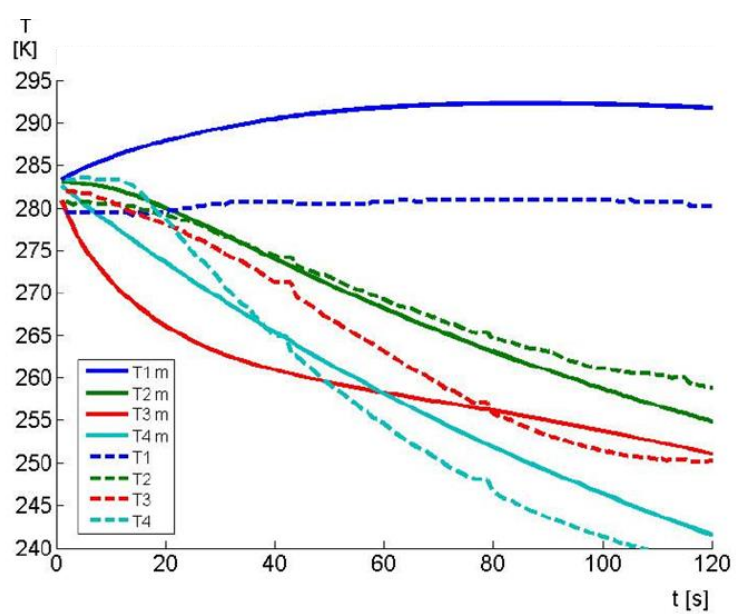

Fig. 3. Comparison of model temperature changes with experimental results for outflow diameter $d=6 \mathrm{~mm}$.

According to the above results, only the temperature of the upper part of the tank $\left(T_{1}\right)$ increases during the jet fire. This is the result of the thermal radiation of the flame (with an initial stream value $<750 \mathrm{~W}$ ) only on this part of the tank when the outflow is directed vertically upwards. The lowest values are obtained for the temperature of the liquid phase $\left(\mathrm{T}_{4}\right)$, and therefore the heat required to evaporate the liquid phase is high. The temperature of the lower part of the tank $\left(\mathrm{T}_{2}\right)$ decreases in a similar way to the temperature of the liquid phase. The decrease in $T_{2}$ is slightly smaller than $\mathrm{T}_{4}$. The liquid phase absorbs large amounts of heat from the lower part of the shell of the tank. A slightly different course is characterized by gas phase temperature $\left(T_{3}\right)$. Initially, its drop is more rapid than in the case of the liquid phase, then it becomes milder and finally, the temperature of the liquid phase is much lower. The rapid decrease in the initial phase is the result of the discharge of a large amount of heat from the gas phase to the environment. As the time passes, the pressure in the tank drops, so that the flow and speed of the outflow are also reduced.

Temperature measurements carried out during the jet fire tests for outflow through a diameter of $6 \mathrm{~mm}$ and an ambient temperature of approx. 280-285K confirm the tendency of temperature changes obtained in the model. In the case of the temperature of the upper part of the tank $\left(\mathrm{T}_{1}\right)$, an increase of approx. $10 \mathrm{~K}$ was noted, which is not confirmed by experimental studies. The difference is due to the fact that the calculation assumes that the flame during combustion is stable, its shape does not change and the influence of wind is excluded [5, 13-17]. The temperature of the lower part of the tank $\left(\mathrm{T}_{2}\right)$ obtained as a result of the simulation is very similar to the temperature measured in reality. The model temperature of the liquid phase $\left(\mathrm{T}_{4}\right)$ maintains a similar trend to the temperature of the liquid phase during the experimental test. The temperature $\mathrm{T}_{4}$ obtained from the model is characterized by a constant decrease in time, but in experimental measurement, the drop in temperature $\mathrm{T}_{4}$ in the first minute is more distinct than in the subsequent time of the experiment. In the case of the gas phase temperature $\left(T_{3}\right)$, convergence in the simulation and actual results were also 
achieved, however, in the initial phase a difference in the course of these temperatures is visible. The analysis of calculations in the model was adopted for the simulation time of $120 \mathrm{~s}$. The model includes only transition boiling, film boiling was not included. During the film boiling, the evaporation heat flux

decreases significantly. Hence, after reaching the appropriate difference between the temperature of the liquid and the saturation temperature, the temperature drop of the liquid phase slows down and as a consequence the drop of temperatures $T_{1}, T_{2}$ slows down as well and even the increase of the gas phase temperature appears.

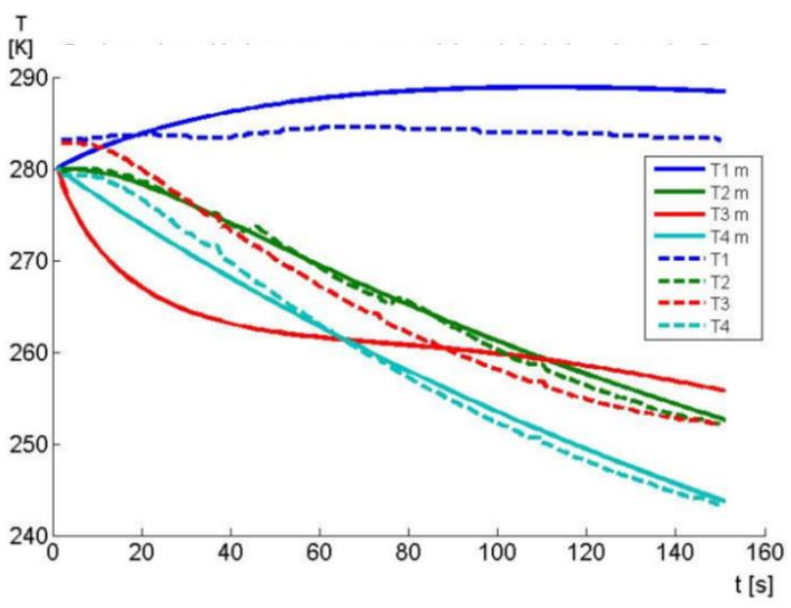

Fig. 4. Comparison of model temperature changes with experimental results for outflow diameter $d=5 \mathrm{~mm}$.

Experimental temperatures for outflow through a diameter of $5 \mathrm{~mm}$ and an ambient temperature of approx. 280-285K also confirm the tendency of temperature changes obtained in the model. For this outflow, the results obtained from the model are even more similar to the empirical results. The temperatures of the liquid phase $\left(\mathrm{T}_{4}\right)$ and the upper part of the tank $\left(\mathrm{T}_{2}\right)$ are almost identical throughout the simulation. The temperature of the gas phase $\left(\mathrm{T}_{3}\right)$ obtained as a result of the simulation temporarily differs significantly from the values actually measured, however, it should be noted that the general trend is maintained, and also the temperature values in the final simulation phase are similar. The model temperature of the upper part of the tank $\left(T_{1}\right)$ as well as in Figure 3 is also characterized by an upward tendency, while the temperature $\mathrm{T}_{1}$ during the experiment remains rather unchanged.

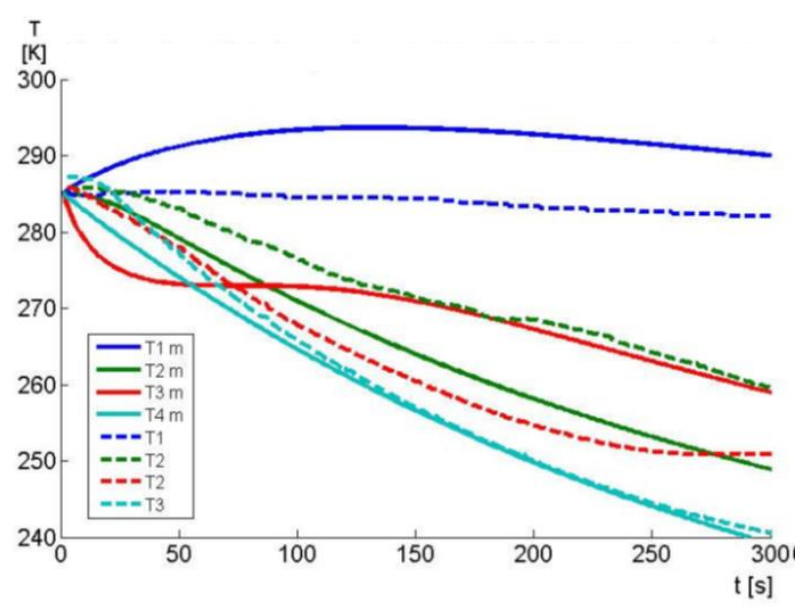

Fig. 5. Comparison of model temperature changes with experimental results for outflow diameter $\mathrm{d}=4 \mathrm{~mm}$.

When comparing the results obtained by the Matlab software with experimental results for a $4 \mathrm{~mm}$ diameter outflow, it can be seen that the liquid phase temperatures $\left(\mathrm{T}_{4}\right)$ in both cases are very similar throughout the simulation. The experimental temperature of the lower part of the tank $\left(\mathrm{T}_{2}\right)$, differs from calculations. Influence of external factors, such as air humidity or wind speed might explain those differences. The temperature of the upper part of the tank $\left(\mathrm{T}_{1}\right)$ does not differ from the previous cases. As a result of the calculations, a higher temperature was obtained. In the case of gas phase temperature $\left(T_{3}\right)$, the differences between calculations and actual results are more visible.

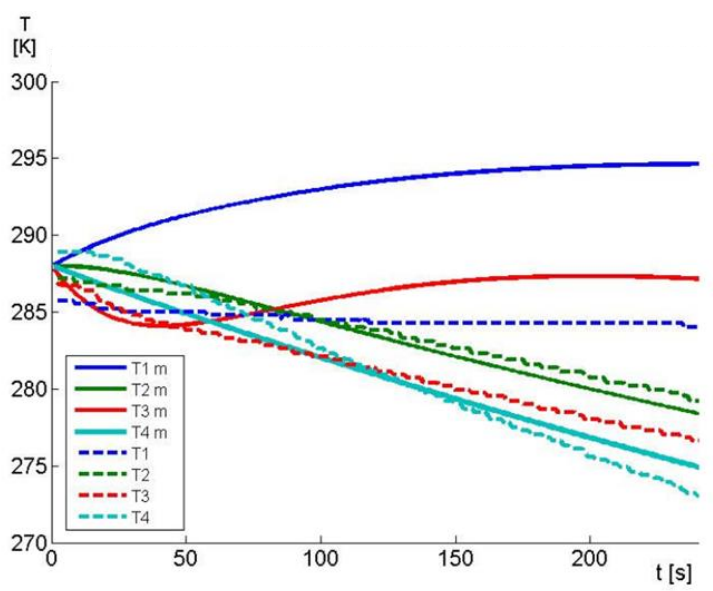

Fig. 6. Comparison of model temperature changes with experimental results for outflow diameter $d=2 \mathrm{~mm}$.

In case of $2 \mathrm{~mm}$ diameter outflow, it can be observed again that the temperature of the liquid phase $\left(\mathrm{T}_{4}\right)$ and the temperature of the lower part of the tank $\left(\mathrm{T}_{2}\right)$ obtained during the simulation is very similar to the experimental results. However, a significant difference was noted for the gas phase temperature $\left(T_{3}\right)$ and the temperature of the upper part of the tank $\left(T_{1}\right)$. The calculated temperature $T_{1}$ in the final phase is about $10 \mathrm{~K}$ higher than the actual temperature, which is a big difference but comparable to the previous results. Although the temperature of the gas 
phase $\left(T_{3}\right)$ obtained as a result of the simulation exceeds the temperature $T_{3}$ measured during the experiment also by about $10 \mathrm{~K}$, however, it is a very big difference compared to the results for larger diameters. In the case of a $2 \mathrm{~mm}$ outflow, the jet was much smaller than in the previous tests, and therefore the evaporation rate was also smaller, as a result of which less heat was absorbed for the liquid evaporation. In the experiment, temperatures $T_{4}$ and $T_{2}$ decreased slower than in previous cases. As a result of a significant increase in temperature $T_{1}$ during the simulation, an excessive increase in temperature $T_{3}$ was obtained in comparison to the experimental results.

The results of the temperature change of the liquid phase $\left(\mathrm{T}_{4}\right)$ obtained in the model are very similar to the experimental data. In the experiment, during the first seconds, the temperature stays at a constant level, after which in the initial phase the drop is more rapid and in the final stage it is milder. The temperature of the lower part of the tank $\left(\mathrm{T}_{2}\right)$ looks similar. In the case of gas phase temperature $\left(T_{3}\right)$, the results differ more. The downward trend is maintained and in the final phase the temperature difference is insignificant, however, in the initial phase, the model has a much faster temperature drop, while in the final part it is slower. In the case of the upper tank temperature $\left(T_{1}\right)$, the difference between the model and the experiment is the most visible. In the model, the temperature increases by less than $10 \mathrm{~K}$, while in the experiment the temperature is rather constant. The model assumes that the flame is stable with a fixed shape and wind speed is not taken into account. In fact, the heat flux coming from the fire was smaller than the one used in the model.

\section{Conclusions}

Taking into account the obtained results of computational simulations and comparing them with the results of experimental research, the first conclusion concerns the temperature course during the jet outflow without ignition. Under these conditions, all analyzed temperatures drop, i.e. the liquid phase, the gas phase, the upper part of the tank's shell and the lower part of the tank's shell. The temperature of the liquid phase decreases the most, while the decrease in the temperature of the upper part of the tank is the smallest. The drop in temperature of the liquid phase results from a large amount of heat necessary to evaporate the liquid phase. Consequently, the temperature of the lower part of the tank and the temperature of the gas phase is also significantly reduced. As the time passes, the evaporation stream decreases, therefore less heat is required to evaporate the liquid phase, as a result, the temperature of the liquid phase and the lower part of the tank are stabilized, and the temperature of the gas phase even begins to grow slightly. The heat required for evaporation has the greatest effect on the temperature distribution in the system. The first impression while observing the phenomenon of jet fire is that the situation should change dramatically in comparison to the jet outflow without ignition. In practice, it turns out that it is not. The temperature of the liquid phase, the temperature of the gas phase and the temperature of the lower part of the tank are still decreasing. Only the temperature of the upper part of the tank remains more or less constant, but it also does not grow. The thermal radiation from the flame only affects the temperature of the upper part of the tank.

The proposed model of the jet outflow explains the phenomena occurring during a jet fire by applying the principles of thermodynamics and the criterion equations. According to the results obtained from simulations carried out in the Matlab computing environment, the temperature of the liquid phase drops the most during the jet outflow. The slightly smaller decrease is recorded for the temperature of the lower part of the tank and the temperature of the gas phase. The temperature of the upper part of the tank decreases in the smallest degree. During a jet fire, the course of changes in the temperature of the liquid phase and the temperature of the lower part of the tank remains almost unchanged. The decrease in the gas phase temperature is slightly slower, while the temperature of the upper part of the tank slightly increases, which differs from the experimental results. The increase in the temperature of the upper part of the tank is the result of the assumptions regarding the stability and constancy of shape of the flame throughout the simulation and the exclusion of the influence of atmospheric conditions. After performing a series of simulations for jet fires at the outflow with different diameters, it can be concluded that as the diameter increases, temperature changes more rapidly, i.e. they decrease more. The most noticeable difference occurs when comparing the temperature of the liquid phase, while the smallest differences are visible for the temperature of the upper part of the tank. The influence of the ambient temperature on the rate of temperature changes is small, but it should be taken into account that assuming that the system is in equilibrium, the initial temperatures of particular phases and parts of the tank are higher. The proposed calculation model allows simulation of various fire conditions.

Summing up, the results obtained by simulations with experimental tests, it was found that the trends regarding temperature changes of the respective phases and part of the tank as well as the mass loss are correct. In addition, the courses of calculation of temperature changes: liquid phase and the lower part of the tank are very similar to real results. Some differences can be seen in the course of the gas phase temperature, while the temperature of the upper part of the tank in each case is greater during simulation. This difference results from the assumptions that the flame is stable and its shape is unchanged in time. The influence of atmospheric conditions, especially wind speed, is also not taken into account. These assumptions also affect the course of changes in the gas phase temperature.

\section{References}

1. M. Bi, J. Ren, B. Zhao, W. Che, Journal of Hazardous Materials 192, p. 874-879 (2011) 
2. X. Zhang, H. Tao, Z. Zhang, J. Liu, X. Liu, Applied Thermal Engineering 132, p. 801-807 (2018)

3. M. Gomez-Mares, M. Zarate, J. Casal, Fire Safety Journal 43(8), p. 583-588 (2009)

4. N. Gopalaswami, Y. Liu, D.M. Laboureur, B. Zhang, M. Sam Mannan, Journal of Loss Prevention in the Process Industries 41, p. 365375 (2016)

5. C. Tao, Y. Shen, R. Zong, Applied Thermal Engineering 91, p. 884-887 (2016)

6. D.M. Laboureur, N. Gopalaswami, Y. Liu, B. Zhang, M. Sam Mannan, Journal of Loss Prevention in the Process Industries 41, p. 355364 (2016)

7. B. Zhang, Y. Liu, D. Laboureur, D. Mannan, Ind. Chem. Res. 54 (37), p. 9251-9256 (2015)

8. P.S. Cumber, M. Spearpoint, Fire Safety Journal 41 (3), p. 215-228 (2006)

9. X.L. Zhang, L.H. Hu, Q. Wang, X.C. Zhang, Y. Jiang, Applied Thermal Engineering 110, p. 11114 2017)

10. I.W. Ekoto, A.J. Ruggles, L.W. Creitz, J.X. Li, Int. J. Hydrogen Energy 39, p. 20570-20577 (2014)

11. K. Zhou, J. Liu, J. Jiang, Applied Thermal Engineering 106, p. 634-639 (2016)

12. K. Zhou, J. Jiang, J. Heat Transfer 138 (2015)

13. M. Gomez-Mares, M. Munoz, J. Casal, Axial temperature distribution in vertical jet fires, J. of Hazardous Materials, 172, p. 54-60 (2009)

14. X. Zhang, L. Hu, W. Zhu, X. Zhang, L. Yang, Applied Thermal Engineering 73, p. 15-22 (2014)

15. A. Palacios, M. Munoz, R.M. Darbra, J. Casal, Fire Safety Journal 51, p. 93-101 (2012)

16. A. Palacios, J. Casal, Fuel 90, p. 824-833 (2011)

17. L. Hu, Q. Wang, F. Tang, M. Delichatsios, X. Zhang, Fuel 106, p. 779-786 (2013)

18. M. Gomez-Mares, M. Munoz, J. Casal, Experimental Thermal and Fluid Science 34, p. 323-329 (2010)

19. D.Y. Krian, D.P. Mishra, Fuel 86, p. 1545-1551 (2007)

20. S.R. Gollahalli, T.A. Brzustowski, H.F. Sullivan, Transactions to the CSME 1975, 3(4), p. 205-214 (1975)

21. Y. A. Cengel, Heat and Mass Transfer, McGraw Hill (2006)
22. H. Clewell, Energy Systems Laboratory, ESLTR-83-03, (1983)

23. P. Cumber, Journal of Hazardous Materials, A89 (2002)

24. Guidelines for Evaluating the Characteristics of Vapor Cloud Explosions, Flash Fires and BLEVE's (American Institute of Chemical Engineers, 1994)

25. J. P. Holman, Heat transfer (McGraw Hill, 1992)

26. D. Incropera, L. Bergman, Fundamentals of Heat and Mass Transfer (Willey, 2006)

27. A. F. Mills, Heat Transfer (Prentice Hall, 1998)

28. R. H. Perry and D. W. Green, Perry's Chemical Engineers' Handbook (The McGraw-Hill Companies, 1999)

29. P. K. Ramskill, The development of ENGULF to model a multi-component liquid in a fire engulfed tank (UKAEA Safety and Reliability Directorate, 1987) 